\title{
The Impact of Diabetes Mellitus and Metformin Treatment on Survival of Patients with Advanced Pancreatic Cancer Undergoing Chemotherapy
}

Younak Choi, MD', Tae-Yong Kim, MD ${ }^{1,2}$, Do-Youn Oh, MD, PhD ${ }^{1,2}$, Kyung-Hun Lee, MD ${ }^{1,2}$, Sae-Won Han, MD, PhD',2, Seock-Ah Im, MD, PhD',2, Tae-You Kim, MD, PhD ${ }^{1,2}$, Yung-Jue Bang, MD, PhD ${ }^{1,2}$ ${ }^{1}$ Department of Internal Medicine, Seoul National University Hospital, Seoul, ${ }^{2}$ Cancer Research Institute, Seoul National University College of Medicine, Seoul, Korea

\section{Supplementary Data}

Table of Contents

Supplementary Table 1 2

Supplementary Table 2 
Supplementary Table 1. Clinical characteristics of DM subsets

\begin{tabular}{|c|c|c|c|c|c|}
\hline \multirow{2}{*}{ Characteristic } & & \multicolumn{3}{|c|}{ DM status $(\mathrm{n}=183)$} & \multirow{2}{*}{ p-value ${ }^{a}$} \\
\hline & & Remote-onset $(n=87)$ & Recent-onset ( $n=73$ ) & Subsequent $(n=23)$ & \\
\hline \multirow[t]{2}{*}{ Sex } & Male & $57(65.5)$ & $44(60.3)$ & $13(56.5)$ & \multirow[t]{2}{*}{0.658} \\
\hline & Female & $30(34.5)$ & $29(39.7)$ & $10(43.5)$ & \\
\hline \multirow[t]{2}{*}{ Age (yr) } & $\geq 60$ & $55(63.2)$ & $39(53.4)$ & $15(65.2)$ & \multirow[t]{2}{*}{0.381} \\
\hline & $<60$ & $32(36.8)$ & $34(46.6)$ & $8(34.8)$ & \\
\hline \multirow[t]{2}{*}{ ECOG PS } & $0-1$ & $69(79.3)$ & $58(79.5)$ & $20(87.0)$ & \multirow[t]{2}{*}{0.694} \\
\hline & $\geq 2$ & $18(20.7)$ & $15(20.5)$ & $3(13.0)$ & \\
\hline \multirow[t]{2}{*}{ Cancer extent } & LAPC & $18(20.7)$ & $16(21.9)$ & $3(13.0)$ & \multirow[t]{2}{*}{0.645} \\
\hline & $\mathrm{MPC}$ & $69(79.3)$ & $57(78.1)$ & $20(87.0)$ & \\
\hline \multirow[t]{3}{*}{ Initial BMI $\left(\mathrm{kg} / \mathrm{m}^{2}\right)$} & $<22.5$ & $52(60.5)$ & $43(58.9)$ & $16(69.6)$ & \multirow[t]{3}{*}{0.153} \\
\hline & $22.5-24.9$ & $26(30.2)$ & $15(20.5)$ & $3(13.0)$ & \\
\hline & $\geq 25.0$ & $8(9.3)$ & $15(20.5)$ & $4(17.4)$ & \\
\hline \multirow{2}{*}{$\begin{array}{l}\text { Weight loss, initial } \\
\text { by BMI } \geq 1 \mathrm{~kg} / \mathrm{m}^{2}\end{array}$} & Yes & $56(64.4)$ & $39(53.4)$ & $15(65.2)$ & \multirow[t]{2}{*}{0.322} \\
\hline & No & $31(35.6)$ & $34(46.6)$ & $8(34.8)$ & \\
\hline \multirow{2}{*}{$\begin{array}{l}\text { Weight loss, during first-line, } \\
\text { by BMI } \geq 1 \mathrm{~kg} / \mathrm{m}^{2}\end{array}$} & Yes & $22(31.4)$ & $21(32.8)$ & $8(40.0)$ & \multirow[t]{2}{*}{0.771} \\
\hline & No & $48(68.6)$ & $43(67.2)$ & $12(60.0)$ & \\
\hline \multirow[t]{2}{*}{ Antidiabetic medication } & Yes & $72(82.8)$ & $51(69.9)$ & $11(47.8)$ & \multirow[t]{2}{*}{0.002} \\
\hline & No & $15(17.2)$ & $22(30.1)$ & $12(52.2)$ & \\
\hline \multirow[t]{2}{*}{ Metformin } & Yes & $28(32.2)$ & $25(34.2)$ & $3(13.0)$ & \multirow[t]{2}{*}{0.142} \\
\hline & No & $59(67.8)$ & $48(65.8)$ & $20(87.0)$ & \\
\hline \multirow[t]{2}{*}{ Sulfonylurea } & Yes & $30(34.5)$ & $27(37.0)$ & $5(21.7)$ & \multirow[t]{2}{*}{0.398} \\
\hline & No & $57(65.5)$ & $46(63.0)$ & $18(78.3)$ & \\
\hline \multirow[t]{2}{*}{ Insulin } & Yes & $30(34.5)$ & $13(17.8)$ & 0 & \multirow[t]{2}{*}{0.001} \\
\hline & No & $57(65.5)$ & $60(82.2)$ & $23(100)$ & \\
\hline \multirow[t]{2}{*}{ CA19-9 } & Elevated & $71(81.6)$ & $63(86.3)$ & $15(65.2)$ & \multirow[t]{2}{*}{0.076} \\
\hline & Normal & $16(18.4)$ & $10(13.7)$ & $8(34.8)$ & \\
\hline \multirow[t]{2}{*}{ Albumin } & Decreased & $20(23.0)$ & $21(28.8)$ & $5(21.7)$ & \multirow[t]{2}{*}{0.649} \\
\hline & Normal & $67(77.0)$ & $52(71.2)$ & $18(78.3)$ & \\
\hline \multirow[t]{2}{*}{ Response } & Controlled & $51(58.6)$ & $43(58.9)$ & $14(60.9)$ & 0.981 \\
\hline & Uncontrolled & $36(41.4)$ & $30(41.1)$ & $9(39.1)$ & \\
\hline Courses of chemotherapy & 1 & $41(47.1)$ & $35(47.9)$ & $14(60.9)$ & 0.485 \\
\hline & $\geq 2$ & $46(52.9)$ & $38(52.1)$ & $9(39.1)$ & \\
\hline
\end{tabular}

Values are presented as number (\%). DM, diabetes mellitus; ECOG PS, Eastern Cooperative Oncology Group performance status; LAPC, locally advanced pancreatic cancer; MPC, metastatic pancreatic cancer; BMI, body mass index. ${ }^{\text {a) Fisher exact }}$ test. 
Supplementary Table 2. Clinical characteristics of DM patients, with/without metformin use

\begin{tabular}{|c|c|c|c|c|}
\hline \multirow{2}{*}{ Characteristic } & & \multicolumn{2}{|c|}{ Metformin vs. No metformin $(n=183)$} & \multirow{2}{*}{ p-value ${ }^{a)}$} \\
\hline & & With metformin $(n=56)$ & Without metformin $(n=127)$ & \\
\hline \multirow[t]{2}{*}{ Sex } & Male & $32(57.1)$ & $82(64.6)$ & \multirow[t]{2}{*}{0.408} \\
\hline & Female & $24(42.9)$ & $45(35.4)$ & \\
\hline \multirow[t]{2}{*}{ Age (yr) } & $\geq 60$ & $39(69.6)$ & $70(55.1)$ & \multirow[t]{2}{*}{0.074} \\
\hline & $<60$ & $17(30.4)$ & $57(44.9)$ & \\
\hline \multirow[t]{2}{*}{ ECOG PS } & $0-1$ & $48(85.7)$ & $99(78.0)$ & \multirow[t]{2}{*}{0.237} \\
\hline & $\geq 2$ & $8(14.3)$ & $28(22.0)$ & \\
\hline \multirow[t]{2}{*}{ Cancer extent } & LAPC & $14(25.0)$ & $23(18.1)$ & \multirow[t]{2}{*}{0.320} \\
\hline & MPC & $42(75.0)$ & $104(81.9)$ & \\
\hline \multirow[t]{3}{*}{ Initial BMI $\left(\mathrm{kg} / \mathrm{m}^{2}\right)$} & $<22.5$ & $31(55.4)$ & $80(63.5)$ & \multirow[t]{3}{*}{0.253} \\
\hline & $22.5-24.9$ & $18(32.1)$ & $26(20.6)$ & \\
\hline & $\geq 25.0$ & $7(12.5)$ & $20(15.9)$ & \\
\hline \multirow{2}{*}{$\begin{array}{l}\text { Weight loss, initial } \\
\text { by } \mathrm{BMI} \geq 1 \mathrm{~kg} / \mathrm{m}^{2}\end{array}$} & Yes & $34(60.7)$ & $76(59.8)$ & \multirow{2}{*}{$>0.999$} \\
\hline & No & $22(39.3)$ & $51(40.2)$ & \\
\hline \multirow{2}{*}{$\begin{array}{l}\text { Weight loss, during first-line, } \\
\text { by } \mathrm{BMI} \geq 1 \mathrm{~kg} / \mathrm{m}^{2}\end{array}$} & Yes & $14(30.4)$ & $37(34.3)$ & \multirow[t]{2}{*}{0.711} \\
\hline & No & $32(69.6)$ & $71(65.7)$ & \\
\hline \multirow[t]{2}{*}{ Sulfonylurea } & Yes & $29(51.8)$ & $33(26.0)$ & \multirow[t]{2}{*}{0.001} \\
\hline & No & $27(48.2)$ & $94(74.0)$ & \\
\hline \multirow[t]{2}{*}{ Insulin } & Yes & $6(10.7)$ & $37(29.1)$ & \multirow[t]{2}{*}{0.008} \\
\hline & No & $50(89.3)$ & $90(70.9)$ & \\
\hline \multirow[t]{2}{*}{ CA19-9 } & Elevated & $45(80.4)$ & $104(81.9)$ & \multirow[t]{2}{*}{0.838} \\
\hline & Normal & $11(19.6)$ & $23(18.1)$ & \\
\hline \multirow[t]{2}{*}{ Albumin } & Decreased & $13(23.2)$ & $33(26.0)$ & \multirow[t]{2}{*}{0.717} \\
\hline & Normal & $43(76.8)$ & $94(74.0)$ & \\
\hline \multirow[t]{2}{*}{ Response } & Controlled & $36(64.3)$ & $72(56.7)$ & \multirow[t]{2}{*}{0.415} \\
\hline & Uncontrolled & $20(35.7)$ & $55(43.3)$ & \\
\hline \multirow[t]{2}{*}{ Courses of chemotherapy } & 1 & $28(50.0)$ & $62(48.8)$ & \multirow[t]{2}{*}{$>0.999$} \\
\hline & $\geq 2$ & $28(50.0)$ & $65(51.2)$ & \\
\hline
\end{tabular}

Values are presented as number (\%). DM, diabetes mellitus; ECOG PS, Eastern Cooperative Oncology Group performance status; LAPC, locally advanced pancreatic cancer; MPC, metastatic pancreatic cancer; BMI, body mass index; CA19-9, cancer

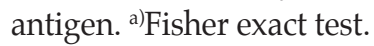


Supplementary Table 3. Metformin recipients vs. all other patients

\begin{tabular}{|c|c|c|c|c|}
\hline \multirow{2}{*}{ Characteristic } & & \multicolumn{2}{|c|}{ Metformin vs. All others $(n=349)$} & \multirow{2}{*}{ p-value ${ }^{a)}$} \\
\hline & & Metformin $(\mathrm{n}=56)$ & All others $(n=293)$ & \\
\hline \multirow[t]{2}{*}{ Sex } & Male & $32(57.1)$ & $192(65.5)$ & \multirow[t]{2}{*}{0.287} \\
\hline & Female & $24(42.9)$ & $101(34.5)$ & \\
\hline \multirow[t]{2}{*}{ Age (yr) } & $\geq 60$ & $39(69.6)$ & $133(45.4)$ & \multirow[t]{2}{*}{0.001} \\
\hline & $<60$ & $17(30.4)$ & $160(54.6)$ & \\
\hline \multirow[t]{2}{*}{ ECOG PS } & $0-1$ & $48(85.7)$ & $233(79.5)$ & \multirow[t]{2}{*}{0.358} \\
\hline & $\geq 2$ & $8(14.3)$ & $60(20.5)$ & \\
\hline \multirow[t]{2}{*}{ Cancer extent } & LAPC & $14(25.0)$ & $52(17.7)$ & \multirow[t]{2}{*}{0.263} \\
\hline & MPC & $42(75.0)$ & $241(82.3)$ & \\
\hline \multirow[t]{3}{*}{ Initial BMI $\left(\mathrm{kg} / \mathrm{m}^{2}\right)$} & $<22.5$ & $31(55.4)$ & $183(63.3)$ & \multirow[t]{3}{*}{0.476} \\
\hline & $22.5-24.9$ & $18(32.1)$ & $71(24.6)$ & \\
\hline & $\geq 25.0$ & $7(12.5)$ & $35(12.1)$ & \\
\hline \multirow{2}{*}{$\begin{array}{l}\text { Weight loss, initial } \\
\text { by BMI } \geq 1 \mathrm{~kg} / \mathrm{m}^{2}\end{array}$} & Yes & $34(60.7)$ & $159(54.3)$ & \multirow[t]{2}{*}{0.384} \\
\hline & No & $22(39.3)$ & $134(45.7)$ & \\
\hline \multirow{2}{*}{$\begin{array}{l}\text { Weight loss, during first-line, } \\
\text { by BMI } \geq 1 \mathrm{~kg} / \mathrm{m}^{2}\end{array}$} & Yes & $14(30.4)$ & $82(33.6)$ & \multirow[t]{2}{*}{0.735} \\
\hline & No & $32(69.6)$ & $162(66.4)$ & \\
\hline \multirow[t]{2}{*}{ CA19-9 } & Elevated & 45 (80.4) & $237(80.9)$ & \multirow[t]{2}{*}{$>0.999$} \\
\hline & Normal & 11 (19.6) & $56(19.1)$ & \\
\hline \multirow[t]{2}{*}{ Albumin } & Decreased & $13(23.2)$ & $69(23.5)$ & \multirow[t]{2}{*}{$>0.999$} \\
\hline & Normal & $43(76.8)$ & $224(76.5)$ & \\
\hline \multirow[t]{2}{*}{ Response } & Controlled & $36(64.3)$ & $161(54.9)$ & \multirow[t]{2}{*}{0.240} \\
\hline & Uncontrolled & $20(35.7)$ & $132(45.1)$ & \\
\hline \multirow[t]{2}{*}{ Courses of chemotherapy } & 1 & $28(50.0)$ & $157(53.6)$ & \multirow[t]{2}{*}{0.663} \\
\hline & $\geq 2$ & $28(50.0)$ & $136(46.4)$ & \\
\hline
\end{tabular}

Values are presented as number (\%). ECOG PS, Eastern Cooperative Oncology Group Performance Status; LAPC, locally advanced pancreatic cancer; MPC, metastatic pancreatic cancer; BMI, body mass index; CA19-9, cancer antigen. ${ }^{\text {a) Fisher exact }}$ test. 